\title{
NODAL SOLUTIONS FOR FOURTH ORDER ELLIPTIC EQUATIONS WITH CRITICAL EXPONENT ON COMPACT MANIFOLDS
}

\author{
MOHAMED BEKIRI AND MOHAMMED BENALILI
}

\begin{abstract}
Using a variational method, we prove the existence of nodal solutions to prescribed scalar $Q$ - curvature type equations on compact Riemannian manifolds with boundary. These equations are fourth-order elliptic equations with critical Sobolev growth .
\end{abstract}

\section{INTRODUCTION}

The Paneitz operator was discovered by Paneitz on 4-dimension manifolds ( see [23]) and extended by Branson to higher dimensions $(n \geq 5)$ ( see [5]) is given by

$$
P_{g}^{n}(u)=\Delta_{g}^{2} u-\operatorname{div}_{g}\left(\frac{(n-2)^{2}+4}{2(n-1)(n-2)} R_{g} g-\frac{4}{n-2} R_{i c}\right)^{\#} d u+\frac{n-4}{2} Q_{g}^{n} u
$$

where $\Delta_{g} u=-\operatorname{div}_{g}(\nabla u)$ is the Laplace-Beltrami operator, $R_{g}$ and $\operatorname{Ric}_{g}$ denote the scalar curvature and Ricci curvature respectively, the symbol \# stands for the musical isomorphism and $Q_{g}^{n}$ is the $Q$-curvature which is expressed by

$$
Q_{g}^{n}=\frac{1}{2(n-1)} \Delta_{g} R+\frac{n^{3}-4 n^{2}+16 n-16}{8(n-1)^{2}(n-2)^{2}} R^{2}-\frac{2}{(n-2)^{2}}\left|R i c_{g}\right|^{2} .
$$

Geometrically the $Q$-curvature can be interpreted as the analogue of the scalar curvature for the conformal Laplacian.

Let $(M, g)$ be a smooth Riemannian compact manifold with boundary and of dimension $(n \geq 5)$. We let $A$ be a smooth symmetric $(2,0)$-tensor on $M$ and $a \in C^{\infty}(M)$. The Paneitz-Branson type operator with general coefficients is an operator of the form

$$
P_{g} u=\Delta_{g}^{2} u-\operatorname{div}_{g}\left(A(\nabla u)^{\#}\right)+a u \text {. }
$$

Date: October 22, 2016.

2000 Mathematics Subject Classification. Primary 58J05.

Key words and phrases. Fourth elliptic equation, Nodal solutions, Critical Sobolev exposent. 
If the coefficients are constant it reads as follows

$$
P_{g}^{n} u=\Delta_{g}^{2} u+\alpha \Delta_{g} u+\beta u .
$$

In particular on Einstein manifolds, it is reduced to

$$
P_{g}^{n}(u)=\Delta_{g}^{2} u+\alpha_{n} \Delta_{g} u+\beta_{n} u
$$

where

$$
\alpha_{n}=\frac{n^{2}-2 n-4}{2 n(n-1)} R_{g} \text { and } \beta_{n}=\frac{(n-4)\left(n^{2}-4\right)}{16(n-1)^{2}} R_{g}^{2}
$$

A nice property of the Paneitz-Branson operator is its conformal covariance i.e. if $\tilde{g}=\varphi^{\frac{4}{n-4}} g$ is a conformal metric to $g$ where $\varphi$ stands for a smooth positive function, then for all $u \in C^{\infty}(M)$ we have

$$
P_{g}^{n}(u \varphi)=\varphi^{\frac{n+4}{n-4}} P_{\tilde{g}}^{n}(u) .
$$

In particular, by setting $u=1$, we obtain

$$
P_{g}^{n} \varphi=\frac{n-4}{2} Q_{\tilde{g}}^{n} \varphi^{\frac{n+4}{n-4}}
$$

In this work seek the existence of a real number $\lambda$ and a nodal solution $u$ of the following Dirichlet problem

$$
\begin{cases}P_{g} u=\lambda f|u|^{2^{\sharp}}-2 & \text { in } M \\ u=\phi_{1}, \partial_{\nu} u=\phi_{2} & \text { on } \partial M\end{cases}
$$

where $P_{g}$ is the Paneitz-Branson type operator defined by (1.2), $\phi_{1}, \phi_{2} \in$ $C^{\infty}(\partial M)$ are boundary data and $2^{\sharp}=\frac{2 n}{n-4}$ is the Sobolev critical exponent. When $\phi_{1}$ changes sign, $u$ is called a nodal solution of the equation (1.4).

Many authors have paid attention to the study of nodal solutions to the second-order Dirichlet problem and several works were carried out; we quote some of them :

In 1990, F.V. Atkinson, H. Brezis and L.A. Peletier [1, studied the existence of nodal solutions of the following eigenvalue problem

$$
\begin{cases}-\Delta u=\lambda u+|u|^{p} u & \text { in } B \\ u \neq \equiv 0 & \text { in } B \\ u=0 & \text { on } \partial B\end{cases}
$$

where $B$ is the unit ball of $\mathbb{R}^{n}(n \geq 3), p=\frac{n+2}{n-2}$, and $\lambda$ is a positive real number.

In 1994, E. Hebey and M. Vaugon [18] obtained existence and multiplicity results of nodal solutions to the problem

$$
\begin{cases}-\Delta_{g} u+a u=f|u|^{\frac{4}{n-2}} u & \text { in } \Omega \\ u \neq \equiv 0 & \text { in } \Omega \\ u=0 & \text { on } \partial \Omega\end{cases}
$$

where $\Omega$ is a smooth bounded domain of $R^{n}(n \geq 3), a, f \in C^{\infty}(\bar{\Omega})$ and $g$ is a Riemannian metric defined in a neighborhood of $\bar{\Omega}$. Moreover, they 
considered this problem when the zero data on the boundary is replaced by a non-zero one.

In [19] D. Holcman obtained by variational method nodal solutions to the following problem

$$
\begin{cases}\Delta_{g} u+a u=\lambda f|u|^{\frac{4}{n-2}} u & \text { in } M \\ u=\phi & \text { on } \partial M\end{cases}
$$

where $(M, g)$ is a smooth compact Riemannian manifold with boundary and of dimension $n \geq 3, a, f \in C^{\infty}(M), \phi \in C^{\infty}(\partial M)$ is a changing sign function and where $\lambda$ stands for a real positive number.

In 2002, Z. Djadli and A. Jourdain [11] studied nodal solutions for scalar curvature type equations with perturbations.

With the discovery of the Paneitz invariant and consequently the introduction of the Q-curvature studies similar to those conducted for the scalar curvature were undertaken by several people. The latter consists in the search for solutions to fourth-order elliptic equations with critical exponents in the sense of the Sobolev inclusions. We refer the reader to the works by Benalili [4, Chang [8, Chang-Yang [9], Djadli-Hebey-Ledoux [10, Djadli-Malchiodi-Ould Ahmedou [12, [13, Esposito-Robert [15], Felli [16], Hebey-Robert [17, Lin [21, Robert [24, 25], to quote only few of them.

We denote by $H_{2,0}^{2}(M)$ the standard Sobolev space which is the completion of the space $C_{c}^{\infty}(M)$ with respect to the norm

$$
\|u\|_{H_{2}^{2}(M)}^{2}=\sum_{i=0}^{i=2}\left\|\nabla^{i} u\right\|_{2}^{2}
$$

where $C_{c}^{\infty}(M)$ denotes the space of smooth functions with compact supports in $M$.

This standard is equivalent to the following Hilbert norm

$$
\|u\|^{2}=\|\Delta u\|_{2}^{2}+\|\nabla u\|_{2}^{2}+\|u\|_{2}^{2} .
$$

We know from the Sobolev theorem that the inclusion $H_{2,0}^{2}(M)$ is continuously embedded in $L^{2^{\sharp}}(M)$, but it is not compactly embedded. Let $K_{0}$ be the best constant in the Euclidean Sobolev inequality:

$$
\|u\|_{2^{\sharp}}^{2} \leq K_{0}\|\Delta u\|_{2}^{2}
$$

for all $u \in C_{c}^{\infty}(R)$. The value of $K_{0}$ was computed by Edmunds-FortunatoJanelli [14, Lieb [20] and Lions [22]. They obtained that

$$
\frac{1}{K_{0}}=\frac{n\left(n^{2}-4\right)(n-4) \omega_{n}^{\frac{4}{n}}}{16}
$$

where $\omega_{n}$ denotes the volume of the Euclidean unit sphere $\left(\mathbb{S}^{n}, h\right)$, endowed with its standard metric.

Following standard terminology, we say that the Paneitz-Branson type operator $P_{g}=\Delta_{g}^{2}-\operatorname{div}_{g}\left(A(\nabla .)^{\#}\right)+a$ is coercive if there exists $\Lambda>0$, 
such that for any $u \in H_{2,0}^{2}(M)$

$$
\int_{M} u P_{g} u d v_{g} \geq \Lambda\|u\|_{H_{2,0}^{2}(M)}^{2} .
$$

In this paper we extend some results obtained by D. Holcman [19] for equations of the Yamabe type to the equations containing the operator of the Paneitz-Branson type. The main results of our study are summarized in the following theorem which is the version with boundary of Theorem 3 of Episoto-Robert [15].

Theorem 1. Let $(M, g)$ be a compact Riemannian manifold of dimension $n \geq 6$ with boundary $\partial M \neq \emptyset$. Let $A$ be a smooth symmetric $(2,0)$-tensor and $a, f \in C^{\infty}(M), f>0$ and $x_{0} \in M$ such that $f\left(x_{0}\right)=\max _{M} f$. We assume that the operator $P_{g}=\Delta_{g}^{2}-\operatorname{div}_{g}\left(A(\nabla .)^{\#}\right)+a$ is coercive and

$8(n-1) \operatorname{Tr}_{g} A\left(x_{0}\right)+(n-6)(n-4)(n+2) \frac{\Delta f\left(x_{0}\right)}{f\left(x_{0}\right)}-4\left(n^{2}-2 n-4\right) R_{g}\left(x_{0}\right)<0$.

Then there exists a positive real number $\lambda$ and a nontrivial solution $w=u-$ $h \in H_{2,0}^{2}(M) \cap C^{4}(M)$ of the equation (1.4) which is a minimizer of the functional I defined on $H_{2,0}^{2}(M)$ by $I(u)=\int_{M}\left(\left(\Delta_{g} u\right)^{2}+A\left((\nabla u)^{\#},(\nabla u)^{\#}\right)+a u^{2}\right) d v_{g}$ under the constraint $\int_{M} f|u+h|^{2^{\sharp}} d v_{g}=\gamma$, where $h$ denotes the unique solution of the problem

$$
\begin{cases}\Delta_{g}^{2} h-\operatorname{div}_{g}\left(A(\nabla h)^{\#}\right)+a h=0 & \text { in } M \\ h=\phi_{1}, \partial_{\nu} h=\phi_{2} & \text { on } \partial M\end{cases}
$$

and where $\phi_{1}, \phi_{2}$ are smooth functions on the boundary $\partial M$ with $\phi_{1}$ is of changing sign.

Remark 1. Since the function $\phi_{1}$ is of changing sign, the solutions obtained in Theorem 1 are nodal.

The proof of theorem 1 proceeds in several steps: in a first section we use the approach developed by H. Yamabe in [27]: we construct a minimizing sequence of solutions to the subcritical equations. In the second one, we show that this sequence converges weakly to a solution of the critical equation when the subcritical exponent tends to the critical Sobolev exponent. In the third section we show that under some conditions, we obtain a non trivial solution of the critical equation. The last section is devoted to test functions which verify the conditions assumed in the generic Proposition ??.

\section{SubCRITICAL SOlutions}

We shall make use of the following Sobolev inequality on compact manifolds with boundary obtained in a more general context (see [6]) 
Lemma 1. ([6]) Let $(M, g)$ be a smooth compact Riemannian manifold with boundary of dimension $n \geq 5$. Then given $\varepsilon>0$, then there exists a positive constant $B$ which depends on $M, g$, $\varepsilon$ such that

$$
\|u\|_{2^{\sharp}}^{2} \leq\left(K_{0}^{2}+\varepsilon\right)\|\Delta u\|_{2}^{2}+B\|u\|_{2}^{2}
$$

for all $u \in H_{2,0}^{2}(M)$.

First, we state the following useful proposition.

Proposition 1. Let $(M, g)$ be a smooth Riemannian compact manifold with smooth boundary and of dimension $n \geq 6$. We assume that the operator $P_{g}$ defined in (1.2) is coercive. Then there exists a unique $h \in C^{4}(M)$ solution of the following problem

$$
\left\{\begin{array}{ll}
P_{g} h=0 & \text { in } M \\
h=\phi_{1}, \partial_{\nu} h=\phi_{2} & \text { on } \partial M
\end{array} .\right.
$$

Proof. Since the operator $P_{g}$ is coercive, the existence and the uniqueness of the solution are guaranteed by the Lax-Milgram's theorem. The regularity follows from general regularity theory.

Let $w=u-h$, we first notice that $u \in C^{4}(M)$ is solution of the equation (1.4) if and only if $w \in C^{4}(M)$ is solution of

$$
\left\{\begin{array}{ll}
P_{g} w=\lambda f|w+h|^{2^{\sharp}-2}(w+h) & \text { in } M \\
w=\partial_{\nu} w=0 & \text { on } \partial M
\end{array} .\right.
$$

The associated subcritical problem to 2.2 is then

$$
\begin{cases}P_{g} w=\lambda f|w+h|^{q-2}(w+h) & \text { in } M \\ w=\partial_{\nu} w=0 & \text { on } \partial M\end{cases}
$$

where $2<q<2^{\sharp}$.

The functional associated to equation (2.3) is defined on $H_{2,0}^{2}(M)$ by

$$
I(w)=\int_{M} w P_{g} w d v_{g}=\int_{M}\left((\Delta w)^{2}+A\left((\nabla w)^{\#},(\nabla w)^{\#}\right)+a w^{2}\right) d v_{g} .
$$

Denote by

$\mu_{\gamma, q}:=\inf _{w \in \mathcal{H}_{q}} I(w)$ and $\mathcal{H}_{q}=\left\{w \in H_{2,0}^{2}(M)\right.$ such that $\left.\int_{M} f|w+h|^{q} d v_{g}=\gamma\right\}$

where $\gamma$ is a constant such that

$$
\int_{M} f|h|^{2^{\sharp}} d v_{g}<\gamma .
$$

We state that

Lemma 2. For all $\gamma>\int_{M} f|h|^{2^{\sharp}} d v_{g}$ and for all $0 \leq q<2^{\sharp}$, there exists a real number $\lambda_{\gamma, q}$ and a function $w_{\gamma, q} \in \mathcal{H}_{q}$ solution of the problem (2.3). 
Proof. First we show that $\mathcal{H}_{q}$ is not empty under the condition $\int_{M} f|h|^{2^{\sharp}} d v_{g}<$ $\gamma$. To do this, we set

$$
F(t)=\int_{M} f\left|t \psi_{1}+h\right|^{q} d v_{g}
$$

where $\psi_{1}$ is the eigenfunction corresponding to the first eigenualue $\lambda_{1}$ of $\Delta_{g}^{2}$ i.e.

$$
\left\{\begin{array}{ll}
\Delta_{g}^{2} \psi_{1}=\lambda_{1} \psi_{1} & \text { in } M \\
\psi_{1}=\partial_{\nu} \psi_{1}=0 & \text { on } \partial M
\end{array} .\right.
$$

It is clear that

$$
F(0)=\int_{M} f|h|^{q} d v_{g}<\gamma
$$

and

$$
\lim _{t \rightarrow+\infty} F(t)=+\infty
$$

Prom the continuity of $F$, there exists $t_{q}>0$ such that

$$
F\left(t_{q}\right)=\int_{M} f\left|t_{q} \psi_{1}+h\right|^{q} d v_{g}=\gamma
$$

So $t_{q} \psi_{1} \in \mathcal{H}_{q}$.

Secondly we check that $\mu_{\gamma, q}$ is finite. Let $u \in H_{2,0}^{2}(M)$. Since the tensor $A$ is smooth, there exists a constant $C>0$ such that

$$
\left|\int_{M} A\left((\nabla u)^{\#},(\nabla u)^{\#}\right) d v_{g}\right| \leq C \int_{M}|\nabla u|^{2} d v_{g} .
$$

By interpolation (see Aubin [3] page 93), for any $\eta>0$ there exists $C(\eta)>0$ such that for any $u \in H_{2,0}^{2}(M)$

$$
\|\nabla u\|_{2}^{2} \leq \eta\|\Delta u\|_{2}^{2}+C(\eta)\|u\|_{2}^{2} .
$$

Plugging (2.6) into (2.5) we get

$$
\left|\int_{M} A\left((\nabla u)^{\#},(\nabla u)^{\#}\right) d v_{g}\right| \leq C(\eta)\|\Delta u\|_{2}^{2}+C^{\prime}(\eta)\|u\|_{2}^{2} .
$$

Considering inequality (2.7) and the expression of $I$, we deduce that

$$
I(u) \geq(1-C(\eta))\|\Delta u\|_{2}^{2}-\left(C^{\prime}(\eta)+\|a\|_{\infty}\right)\|u\|_{2}^{2}
$$

where $\|\cdot\|_{\infty}$ is the supremum norm.

On the other hand, for all $u \in \mathcal{H}_{q}$, by Hölder's inequality we have

$$
\|u\|_{2}^{2} \leq \operatorname{Vol}_{g}(M)^{1-\frac{2}{q}}\left(\left(\min _{M} f\right)^{-\frac{1}{q}} \gamma^{\frac{1}{q}}+\|h\|_{q}\right)^{2} .
$$

Plugging (2.9) in (2.8) and taking $\eta$ small enough, we get

$$
1-C(\eta)=C_{1}>0 \text {. }
$$

So

$$
I(u) \geq C_{1}\|\Delta u\|_{2}^{2}+C_{2}
$$


where $C_{1}$ some positive constant and $C_{2}$ is a constant independent of $u$.

Let $\left(w_{i}\right)$ be a minimizing sequence of the functional $I$ on $\mathcal{H}_{q}$. Hence for $i$ sufficiently large $I\left(w_{i}\right) \leq \mu_{\gamma, q}+1$ and by (2.10) we obtain

$$
\left\|\Delta w_{i}\right\|_{2}^{2} \leq \frac{1}{C_{1}}\left(\mu_{\gamma, q}+1-C_{2}\right) .
$$

From (2.6) and (2.9) we deduce that $\left\|\nabla w_{i}\right\|_{2}^{2}$ and $\left\|w_{i}\right\|_{2}^{2}$ are bounded, and by (2.11) the sequence $\left(w_{i}\right)$ is bounded in $H_{2,0}^{2}(M)$. By the reflexivity of the latter space, there exists subsequence of $\left(w_{i}\right)$ still denoted $\left(w_{i}\right)$ such that

(a) $\quad w_{i} \rightarrow w_{\gamma, q}$ weakly in $H_{2,0}^{2}(M)$

(b) $\quad w_{i} \rightarrow w_{\gamma, q}$ strongly in $H_{1}^{2}(M)$ and $L^{s}(M)$ for all $s<2^{\sharp}$

(c) $\quad\left\|w_{\gamma, q}\right\|_{H_{2,0}^{2}} \leq \liminf _{i}\left\|w_{i}\right\|_{H_{2,0}^{2}}$

Consequently

$$
\begin{aligned}
I\left(w_{\gamma, q}\right) & =\int_{M}\left(\left(\Delta w_{\gamma, q}\right)^{2}+A\left(\left(\nabla w_{\gamma, q}\right)^{\#},\left(\nabla w_{\gamma, q}\right)^{\#}\right)+a w_{\gamma, q}^{2}\right) d v_{g} \\
& \leq \lim _{i} \inf \left\|\Delta w_{i}\right\|_{2}^{2}+\lim _{i} \int_{M} A\left(\left(\nabla w_{i}\right)^{\#},\left(\nabla w_{i}\right)^{\#}\right) d v_{g}+\lim _{i} \int_{M} a w_{i}^{2} d v_{g} \\
& =\lim _{i} I\left(w_{i}\right)=\mu_{\gamma, q}
\end{aligned}
$$

Since

$$
\int_{M} f\left|w_{\gamma, q}+h\right|^{q} d v_{g}=\lim _{i} \int_{M} f\left|w_{i}+h\right|^{q} d v_{g}=\gamma
$$

we obtain

$$
I\left(w_{\gamma, q}\right)=\mu_{\gamma, q}
$$

so $w_{\gamma, q}$ satisfies

$$
\begin{aligned}
& \int_{M}\left(\Delta w_{\gamma, q} \Delta \varphi+A\left(\left(\nabla w_{\gamma, q}\right)^{\#},(\nabla \varphi)^{\#}\right)+a w_{\gamma, q} \varphi\right) d v_{g} \\
& =\lambda_{\gamma, q} \int_{M} f\left|w_{\gamma, q}+h\right|^{q-2}\left(w_{\gamma, q}+h\right) \varphi d v_{g}
\end{aligned}
$$

for any $\varphi \in H_{2,0}^{2}(M)$; where $\lambda_{\gamma, q}$ is the Lagrange multiplier . Hence $w_{\gamma, q}$ is a weak solution of the equation

$$
\begin{cases}\Delta_{g}^{2} w_{\gamma, q}-\operatorname{div} A\left(\nabla w_{\gamma, q}\right)^{\#}+a w_{\gamma, q}=\lambda_{\gamma, q} f\left|w_{\gamma, q}+h\right|^{q-2}\left(w_{\gamma, q}+h\right) & \text { in } M \\ w_{\gamma, q}=\partial_{\nu} w_{\gamma, q}=0 & \text { on } \partial M\end{cases}
$$

Using the boostrap method, we show that $w_{\gamma, q} \in L^{\infty}(M)$, so $P_{g}\left(w_{\gamma, q}\right)=$ $\Delta_{g}^{2} w_{\gamma, q}-\operatorname{div} A\left(\nabla w_{\gamma, q}\right)^{\#}+a w_{\gamma, q} \in L^{s}(M)$ for any $\left(s<2^{\#}\right)$. Since $P_{g}$ is a fourth order elliptic operator, it follows by a well known regularity theorem that $w_{\gamma, q} \in C^{0, \alpha}(M)$, for some $\alpha \in(0,1)$; hence $w_{\gamma, q} \in C^{4, \alpha}(M)$. 


\section{Critical solutions}

In this section we will show the exitence of a non trivial solution of the critical equation (1.4).

Proposition 2. Under the hypothesis $\int_{M} f|h|^{q} d v_{g}<\gamma$, the sequence $\left(w_{\gamma, q}\right)_{q}$ is bounded in $H_{2,0}^{2}(M)$. The Lagrange multipliers $\lambda_{\gamma, q}$ are strictly positive and the sequence $\left(\lambda_{\gamma, q}\right)_{q}$ is bounded when $q$ tends to $2^{\sharp}$.

Proof. Multiplying (2.12) by $w_{\gamma, q}$ and integrating yield

$$
\begin{aligned}
0 \leq \mu_{\gamma, q} & =\int_{M}\left(P_{g} w_{\gamma, q}\right) w_{\gamma, q} d v_{g} \\
& =\lambda_{\gamma, q} \int_{M} f\left|w_{\gamma, q}+h\right|^{q-2}\left(w_{\gamma, q}+h\right) w_{\gamma, q} d v_{g} \\
& =\lambda_{\gamma, q} \int_{M} f\left|w_{\gamma, q}+h\right|^{q-2}\left(w_{\gamma, q}+h\right)\left(w_{\gamma, q}+h-h\right) d v_{g} \\
& =\lambda_{\gamma, q}\left(\gamma-\int_{M} f\left|w_{\gamma, q}+h\right|^{q-2}\left(w_{\gamma, q}+h\right) h d v_{g}\right)
\end{aligned}
$$

By Hölder's inequality we get

$$
\int_{M} f\left|w_{\gamma, q}+h\right|^{q-2}\left(w_{\gamma, q}+h\right) h d v_{g} \leq \gamma^{1-\frac{1}{q}}\left(\int_{M} f|h|^{q}\right)^{\frac{1}{q}}<\gamma .
$$

We deduce that $\lambda_{\gamma, q} \geq 0$. Moreover if $\lambda_{\gamma, q}=0$, then $w_{\gamma, q}=0$. Hence a contradiction with the fact that $w_{\gamma, q} \in \mathcal{H}_{q}$ and $\int_{M} f|h|^{q} d v_{g}<\gamma$.

Now, we prove that the sequence $\left(w_{\gamma, q}\right)_{q}$ is bounded in $H_{2,0}^{2}(M)$.

Let $\psi_{1}$ be an eigenfunction of $\Delta_{g}^{2}$ corresponding to the eigenvalue $\lambda_{1}$ such that

$$
\begin{cases}\Delta_{g}^{2} \psi_{1}=\lambda_{1} \psi_{1} & \text { in } M \\ \psi_{1}=\partial_{\nu} \psi_{1}=0 & \text { on } \partial M \\ \int_{M} \psi_{1}^{2} d v_{g}=1 & \end{cases}
$$

Let

$$
F(t, q)=\int_{M} f\left|t \psi_{1}+h\right|^{q} d v_{g}
$$

In the last section, we obtain $t_{q} \psi_{1} \in \mathcal{H}_{q}$. Hence

$$
F\left(t_{q}, q\right)=\int_{M} f\left|t_{q} \psi_{1}+h\right|^{q} d v_{g}=\gamma .
$$

In the following we show that $\frac{\partial F}{\partial t}\left(t_{q}, q\right) \neq 0$. We proceed by contradiction.

We suppose that $\frac{\partial F}{\partial t}\left(t_{q}, q\right)=0$ and obviously, we have

$$
\begin{aligned}
t_{q} \frac{\partial F}{\partial t}\left(t_{q}, q\right) & =t_{q} \int_{M} f\left|t_{q} \psi_{1}+h\right|^{q-2}\left(t_{q} \psi_{1}+h\right) \psi_{1} d v_{g} \\
& =\int_{M} f\left|t_{q} \psi_{1}+h\right|^{q-2}\left(t_{q} \psi_{1}+h\right)\left(t_{q} \psi_{1}+h-h\right) d v_{g} \\
& =\gamma-\int_{M} f\left|t_{q} \psi_{1}+h\right|^{q-2}\left(t_{q} \psi_{1}+h\right) h d v_{g}=0
\end{aligned}
$$

Then

$$
\gamma=\int_{M} f\left|t_{q} \psi_{1}+h\right|^{q-2}\left(t_{q} \psi_{1}+h\right) h d v_{g}
$$


Using the Hölder's inequality and the hypothesis $\int_{M} f|h|^{q} d v_{g}<\gamma$, we deduce that

$$
\int_{M} f\left|t_{q} \psi_{1}+h\right|^{q-2}\left(t_{q} \psi_{1}+h\right) h d v_{g} \leq\left(\int_{M} f\left|t_{q} \psi_{1}+h\right|^{q} d v_{g}\right)^{1-\frac{1}{q}}\left(\int_{M} f|h|^{q} d v_{g}\right)^{\frac{1}{q}}<\gamma .
$$

which contradicts with (3.1).

Since $\frac{\partial F}{\partial t}\left(t_{q}, q\right) \neq 0$ and from the implicit function theorem, it results that $t_{q}$ is continuous function of $q$. Hence there exists a constant $C(\gamma)$ independent of $q$ such that

$$
\int_{M} w_{\gamma, q} P_{g} w_{\gamma, q} d v_{g} \leq I\left(t_{q} \psi_{1}\right)=t_{q}^{2} I\left(\psi_{1}\right) \leq C(\gamma) .
$$

From the coercivity of $P_{g}$, we find that the sequence $\left(w_{\gamma, q}\right)_{q}$ is bounded in $H_{2,0}^{2}(M)$ when $q$ tends to $2^{\sharp}$.

So, we can extract a subsequence of $\left(w_{\gamma, q}\right)$ still denoted $w_{\gamma, q}$, such that

(a) $\quad w_{\gamma, q} \rightarrow w$ weakly in $H_{2,0}^{2}(M)$ as $q \rightarrow 2^{\sharp}$

(b) $\quad w_{\gamma, q} \rightarrow w$ strongly in $H_{1}^{2}(M)$ and $L^{s}(M)$ for all $s<2^{\sharp}$ as $q \rightarrow 2^{\sharp}$

(c) $w_{\gamma, q} \rightarrow w$ a.e in $M$ as $q \rightarrow 2^{\sharp}$

Now, we prove that the Lagrange multiplier $\lambda_{\gamma, q}$ is bounded when $q$ tends to $2^{\sharp}$.

Using the definition of $\lambda_{\gamma, q}$ and the formula (3.2) and the fact that

$$
\int_{M} f\left|w_{\gamma, q}+h\right|^{q-2}\left(w_{\gamma, q}+h\right) h d v_{g} \leq \gamma^{1-\frac{1}{q}}\left(\int_{M} f|h|^{q} d v_{g}\right)^{\frac{1}{q}}<\gamma
$$

we obtain

$$
\begin{aligned}
0<\lambda_{\gamma, q} & =\frac{\int_{M}\left(P_{g} w_{\gamma, q}\right) w_{\gamma, q} d v_{g}}{\int_{M} f\left|w_{\gamma, q}+h\right|^{q-2}\left(w_{\gamma, q}+h\right) w_{\gamma, q} d v_{g}} \\
& =\frac{\int_{M}\left(P_{g} w_{\gamma, q}\right) w_{\gamma, q} d v_{g}}{\gamma-\int_{M} f\left|w_{\gamma, q}+h\right|^{q-2}\left(w_{\gamma, q}+h\right) h d v_{g}} \\
& \leq \frac{I\left(t_{q} \psi_{1}\right)}{\gamma-\gamma^{1-\frac{1}{q}}\left(\int_{M} f|h|^{q} d v_{g}\right)^{\frac{1}{q}}}<C(\gamma, h) .
\end{aligned}
$$

Since $\left(\lambda_{\gamma, q}\right)_{q}$ is bounded, there is a subsequence of $\left(\lambda_{\gamma, q}\right)_{q}$ still labelled $\left(\lambda_{\gamma, q}\right)_{q}$ which converges to $\lambda$.

Passing to the limit in equation (2.12), we obtain that $w$ the weak limit of the sequence $\left(w_{\gamma, q}\right)_{q}$ is a weak solution of the critical equation (1.4).

Let $u:=w+h$. If $\left(\phi_{1}, \phi_{2}\right) \not \equiv(0,0)$, then $u \not \equiv 0$ is a non trivial solution of the equation

$$
\left\{\begin{array}{ll}
P_{g} u=\lambda f|u|^{2^{\sharp}-2} u & \text { in } M \\
u=\phi_{1} \text { and } \partial_{\nu} u=\phi_{2} & \text { on } \partial M
\end{array} .\right.
$$


And if $\left(\phi_{1}, \phi_{2}\right) \equiv(0,0)$, then $h \equiv 0$ i.e. $u=w$. We will prove that under some condition, $u$ is non trivial solution of the equation

$$
\begin{cases}P_{g} u=\lambda f|u|^{2^{\sharp}}-2 & \text { in } M \\ u=\partial_{\nu} u=0 & \text { on } \partial M\end{cases}
$$

Proposition 3. Suppose that the minimizing sequence $\left(w_{\gamma, q}\right)_{q}$ converges weakly to $w$ and put $\mu=\lim _{q} \mu_{\gamma, q}$. Assume that

$$
\mu<\frac{\gamma^{\frac{2}{2^{\sharp}}}}{K_{0}\|f\|_{\infty}^{\frac{2}{2^{\sharp}}}}
$$

then $w$ is non trivial solution of the equation (3.3).

Proof. First, we have

$$
\gamma^{\frac{2}{q}}=\left(\int_{M} f\left|w_{\gamma, q}\right|^{q}\right)^{\frac{2}{q}} \leq\|f\|_{\infty}^{\frac{2}{q}} \operatorname{Vol}_{g}(M)^{\frac{2}{q}-\frac{2}{2^{\sharp}}}\left(\int_{M}\left|w_{\gamma, q}\right|^{2^{\sharp}}\right)^{\frac{2}{2^{\sharp}}}
$$

Using the Sobolev inequality given by Lemma 1, for any $\epsilon>0$, there exists $B_{\epsilon}>0$ such that

$$
\begin{aligned}
& \gamma^{\frac{2}{q}}\|f\|_{\infty}^{-\frac{2}{q}} V o l_{g}(M)^{\frac{2}{2^{\sharp}}-\frac{2}{q}} \leq\left(K_{0}+\epsilon\right)\left\|\Delta w_{\gamma, q}\right\|_{2}^{2}+B_{\epsilon}\left\|w_{\gamma, q}\right\|_{2}^{2} \\
& \leq\left(K_{0}+\epsilon\right)\left[(1+\bar{\eta})\left\|\Delta w_{\gamma, q}\right\|_{2}^{2}-\bar{\eta}\left\|\Delta w_{\gamma, q}\right\|_{2}^{2}\right]+B_{\epsilon}\left\|w_{\gamma, q}\right\|_{2}^{2}
\end{aligned}
$$

where $\bar{\eta}$ is some small enough constant.

Since

$$
\left\|\Delta w_{\gamma, q}\right\|_{2}^{2}=\mu_{\gamma, q}-\int_{M}\left(A\left(\left(\nabla w_{\gamma, q}\right)^{\#},\left(\nabla w_{\gamma, q}\right)^{\#}\right)+a w_{\gamma, q}^{2}\right) d v_{g}
$$

it follows that

$$
\begin{aligned}
& \gamma^{\frac{2}{q}}\|f\|_{\infty}^{-\frac{2}{q}} \operatorname{Vol}_{g}(M)^{\frac{2}{2^{\sharp}}-\frac{2}{q}} \\
& \leq\left(K_{0}+\epsilon\right)\left\{(1+\bar{\eta})\left[\mu_{\gamma, q}-\int_{M}\left(A\left(\left(\nabla w_{\gamma, q}\right)^{\#},\left(\nabla w_{\gamma, q}\right)^{\#}\right)+a w_{\gamma, q}^{2}\right) d v_{g}\right]-\bar{\eta}\left\|\Delta w_{\gamma, q}\right\|_{2}^{2}\right\}+ \\
& B_{\epsilon}\left\|w_{\gamma, q}\right\|_{2}^{2} .
\end{aligned}
$$

Because $A$ is smooth, then for any $\eta>0$ there exists $C(\eta)>0$ such that

$$
\left|\int_{M} A\left(\left(\nabla w_{\gamma, q}\right)^{\#},\left(\nabla w_{\gamma, q}\right)^{\#}\right) d v_{g}\right| \leq \eta\left\|\Delta w_{\gamma, q}\right\|_{2}^{2}+C(\eta)\left\|w_{\gamma, q}\right\|_{2}^{2}
$$

we obtain

$$
\begin{aligned}
& \gamma^{\frac{2}{q}}\|f\|_{\infty}^{-\frac{2}{q}} V o l_{g}(M)^{\frac{2}{2^{\sharp}}-\frac{2}{q}}-\left(K_{0}+\epsilon\right)(1+\bar{\eta}) \mu_{\gamma, q} \\
& \leq\left(K_{0}+\epsilon\right)\left\{(1+\bar{\eta})\left[\eta\left\|\Delta w_{\gamma, q}\right\|_{2}^{2}+C(\eta)\left\|w_{\gamma, q}\right\|_{2}^{2}+\|a\|_{\infty}\left\|w_{\gamma, q}\right\|_{2}^{2}\right]-\bar{\eta}\left\|\Delta w_{\gamma, q}\right\|_{2}^{2}\right\} \\
& +B_{\epsilon}\left\|w_{\gamma, q}\right\|_{2}^{2}
\end{aligned}
$$


taking $\eta>0$ such that

$$
\bar{\eta}=\frac{\eta}{1-\eta}
$$

we get

$$
\gamma^{\frac{2}{q}}\|f\|_{\infty}^{-\frac{2}{q}} \operatorname{Vol}_{g}(M)^{\frac{2}{2^{\sharp}}-\frac{2}{q}}-\left(K_{0}+\epsilon\right)(1+\bar{\eta}) \mu_{\gamma, q} \leq C(\epsilon, \eta)\left\|w_{\gamma, q}\right\|_{2}^{2}
$$

When $q$ tends to $2^{\sharp}$, the constants $\epsilon, \eta$ are chosen sufficiently small and if

$$
\mu<\frac{\gamma^{\frac{2}{2^{\sharp}}}}{K_{0}\|f\|_{\infty}^{\frac{2}{2^{\sharp}}}}
$$

we infer that

$$
\|w\|_{2}^{2} \geq C^{\prime}>0
$$

Hence $w \not \equiv 0$.

Now we are going to establish the regularity of the solution of equation (2.2). We adapt the technique developed by Van der Vorst [26], DjadliHebey-Ledoux [10] and Esposito-Robert [15], for fourth-order elliptic equation.

First, we will enumerate two facts that will be useful to us. The first one is the boudary version of the theorem given by F. Robert in [25].

Theorem 2. 25] Let $(M, g)$ be a compact Riemannian manifold with boundary of dimension $n \geq 5, a \in C^{\infty}(M)$ and let $A$ be smooth symmetric $(2,0)$ tensor on $M$. Assume that the operator $P_{g}=\Delta_{g}^{2}-\operatorname{div}_{g} A(\nabla .)^{\#}+a$ is a coercive, then for any $f \in H_{k, 0}^{p}(M)$ there exists a unique $u \in H_{k+4,0}^{p}(M)$ such that $P_{g} u=f$. Moreover we have

$$
\|u\|_{H_{k+4,0}^{p}(M)} \leq C\|f\|_{H_{k, 0}^{p}(M)}
$$

Proof. The proof is similar to the one given in [25], we omit it.

Theorem 3. 3] Let $(M, g)$ be a compact Riemannian manifold of dimension $n \geq 1$ and with boundary, $p \geq 1$ and $0 \leq m \leq k$ two integers such that $n \geq p(k-m)$, then $H_{k}^{p}(M)$ is embedded in $H_{m}^{q}(M)$, where $\frac{1}{q}=\frac{1}{p}-\frac{k-m}{n}$.

Our regularity theorem states as follows

Theorem 4. Let $(M, g)$ be a compact Riemannian manifold of dimension $n \geq 5$ and with boundary. Assume that the operator $P_{g}=\Delta_{g}^{2}-\operatorname{div}_{g} A(\nabla .)^{\#}+$ $a$ is coercive. Let $u \in H_{2,0}^{2}(M)$ be a weak solution of equation (2.2), then $u \in C^{4}(M)$ and $u$ is a strong solution of the equation (2.2).

Proof. We follow closely the proof of Episoto-Robert [15] and also that given by Djadli-Hebey-Ledoux [10 The method goes back to Van der Vorst [26]. For any $p>1$, denote by $L_{0}^{p}(M)$ the set of $f \in L^{p}(M)$ with support in $M$. 
Let $u \in H_{2,0}^{2}(M)$ be a weak solution of (2.2) we claim as in [15] that, for any $\epsilon>0$ there exists $q_{\epsilon} \in L_{0}^{\frac{n}{4}}(M), f_{\epsilon} \in L_{0}^{\infty}(M)$ such that

$$
\begin{aligned}
\left(\Delta_{g}+1\right)^{2} u & =\operatorname{div}_{g}\left(A^{\#} d u\right)+(1-a) u+2 \Delta_{g} u+\lambda f|u|^{2^{\sharp}-2} u \\
& =b+q_{\epsilon} u+f_{\epsilon}
\end{aligned}
$$

where $b=\operatorname{div}_{g}\left(A^{\#} d u\right)+(1-a) u+2 \Delta_{g} u$.

According to theorem 2, for any $q>1$ and any $f \in L_{0}^{q}(M)$, there exists a unique $u \in H_{4,0}^{q}(M)$ such that

$$
\left(\Delta_{g}+1\right)^{2} u=f
$$

with

$$
\|u\|_{H_{4}^{q}(M)} \leq\|f\|_{L^{q}(M)} .
$$

Now, we consider the following operator

$$
H_{\epsilon}: u \in L_{0}^{q}(M) \rightarrow\left(\Delta_{g}+1\right)^{-2}\left(q_{\epsilon} u\right) \in L_{0}^{q}(M)
$$

with

$$
\begin{aligned}
\left\|H_{\epsilon} u\right\|_{q} & =O\left(\left\|\left(\Delta_{g}+1\right)^{-2}\left(q_{\epsilon} u\right)\right\|_{q}\right)=O\left(\left\|\left(\Delta_{g}+1\right)^{-2}\left(q_{\epsilon} u\right)\right\|_{H_{4}^{\hat{q}}(M)}\right) \\
& \leq C\left\|q_{\epsilon} u\right\|_{\hat{q}} \leq C\left\|q_{\epsilon}\right\|_{\frac{n}{4}}\|u\|_{q} \leq C \epsilon\|u\|_{q}
\end{aligned}
$$

and $\hat{q}=\frac{n q}{n+4 s}$.

Hence, for $\epsilon>0$ and sufficiently small

$$
\left\|H_{\epsilon}\right\|_{L^{q} \rightarrow L^{q}} \leq C \epsilon<\frac{1}{2}
$$

So, the operator

$$
\begin{aligned}
& \left(I d-H_{\epsilon}\right): L_{0}^{q}(M) \rightarrow L_{0}^{q}(M) \\
& \left(I d-H_{\epsilon}\right) u=\left(\Delta_{g}+1\right)^{-2}\left(b+f_{\epsilon}\right)
\end{aligned}
$$

is an invertible, and we get $b+f_{\epsilon} \in L^{2}(M)$, hence $\left(\Delta_{g}+1\right)^{-2}\left(b+f_{\epsilon}\right) \epsilon$ $H_{4,0}^{2}(M)$.

From the Sobolev theorem, we deduce that

$$
u \in L_{0}^{\frac{2 n}{n-8}}(M), f|u|^{2^{\sharp}-2} u \in L_{0}^{\frac{2 n}{(n-8)\left(2^{\sharp}-1\right)}}(M)=L_{0}^{\frac{2 n(n-4)}{(n-8)(n+4)}}(M) .
$$

Since $\frac{2 n(n-4)}{(n-8)(n+4)}>2$, we obtain

$$
\left(\Delta_{g}+1\right)^{-2} u \in L_{0}^{2}(M) .
$$

We now use a boostrap argument to construct an increasing sequence $\left(p_{i}\right)$ such that $u \in H_{4,0}^{p_{i}}(M)$ for all $i \in \mathbb{N}$

We let $p_{0}=2$, the Sobolev's theorem asserts that

$$
u \in L^{\frac{n p_{i}}{n-4 p_{i}}}(M) \text { and } f|u|^{2^{\sharp}-2} u \in L^{\frac{n p_{i}}{\left(n-4 p_{i}\right)\left(2^{\sharp}-1\right)}}(M)=L^{\frac{n p_{i}(n-4)}{\left(n-4 p_{i}\right)(n+4)}}(M)
$$


then

$$
\left(\Delta_{g}+1\right)^{-2} u \in L_{0}^{p_{i+1}}(M)
$$

where

$$
p_{i+1}=\left\{\begin{array}{cl}
\frac{n p_{i}(n-4)}{\left(n-4 p_{i}\right)(n+4)} & \text { if } p_{i}<\frac{n}{4} \\
+\infty & \text { if } p_{i} \geq \frac{n}{4}
\end{array} .\right.
$$

We can verify by recurrence that for all $i \in \mathbb{N}$ that $p_{i}>\frac{2 n}{n+4}$, hence the sequence $\left(p_{i}\right)_{i}$ is increasing and bounded, consequently it converges to $l \geq 2$ fulfilling the relation

$$
l=\frac{n l(n-4)}{(n-4 l)(n+4)} .
$$

The last equation gives $l=\frac{2 n}{n+4}$ which is a contradiction. Hence $p_{i} \rightarrow+\infty$ and $u \in H_{4,0}^{p}(M)$ for all $p>1$. Applying again the Sobolev's theorem, we get $u \in C^{4}(M)$. Hence $u$ is a strong solution to the critical equation (1.4).

\section{Test FunCtions}

In this section, we prove that the condition (??) in the proposition ?? holds by using test functions.

For this purpose we consider a normal geodesic coordinate system $\left(y^{1}, y^{2}, \ldots, y^{n}\right)$ centred at a point $x_{0}$ where $f$ reaches its maximum. Denote by $S(r)$ the geodesic sphere centred at $x_{0}$ and of radius $r(r<d=$ the injectivity radius)

Let $d \Omega$ be the volume element of the Euclidean unit sphere $S^{n-1}(1)$ and put

$$
G(r)=\frac{1}{\omega_{n-1}} \int_{S(r)} \sqrt{|g(x)|} d \Omega
$$

where $\omega_{n-1}$ is the volume of $S^{n-1}(1)$ and $|g(x)|$ is the determinant of the Riemannian metric $g$.

The Taylor's expansion of $G(r)$ in a neighborhood of $r=0$ is given by

$$
G(r)=1-\frac{R\left(x_{0}\right)}{6 n} r^{2}+o\left(r^{2}\right)
$$

where $R\left(x_{0}\right)$ denotes the scalar curvature of $M$ at $x_{0}$.

Let $B\left(x_{0}, \delta\right)$ be the geodesic ball of radius $\delta$ centred at $x_{0}$ such that $0<$ $2 \delta<d$ and $\eta$ a smooth function given by

$$
\eta(x)=\left\{\begin{array}{ll}
1 & \text { if } x \in B\left(x_{0}, \delta\right) \\
0 & \text { if } x \in M-B\left(x_{0}, 2 \delta\right)
\end{array} .\right.
$$

We consider the following radial smooth function:

$$
u_{\epsilon}(r)=\frac{\eta(r)}{\left(r^{2}+\epsilon^{2}\right)^{\frac{n-4}{2}}}
$$


where $r=d\left(x_{0}, x\right)$ denotes the geodesic distance to the point $x_{0}$. To simplify the computations, for any real positive numbers $p, q$ such that $p-q>1$, we define the following functions, (see [2])

$$
I_{p}^{q}=\int_{0}^{+\infty} \frac{t^{q}}{(1+t)^{p}} d t
$$

The following relations result are immediate

$$
I_{p+1}^{q}=\frac{p-q-1}{p} I_{p}^{q}, \quad I_{p+1}^{q+1}=\frac{q}{p-q-1} I_{p+1}^{q} .
$$

First, we compute the Taylor's expansion of the quotient:

$$
Q_{\epsilon}=\frac{\mu\left(u_{\epsilon}\right)}{\left(\gamma\left(u_{\epsilon}\right)\right)^{\frac{2}{2^{\sharp}}}}
$$

where

$$
\begin{gathered}
\mu\left(u_{\epsilon}\right)=\int_{M}\left(\left(\Delta_{g} u_{\epsilon}\right)^{2}+A^{\#}\left(d u_{\epsilon}, d u_{\epsilon}\right)+a u_{\epsilon}^{2}\right) d v_{g} \\
\gamma\left(u_{\epsilon}\right)=\int_{M} f\left|u_{\epsilon}\right|^{2^{\sharp}} d v_{g} .
\end{gathered}
$$

Now, we will compute each term of the expansion as it has been done [7,

[15] and [25]. In the case $n>6$, we get

$$
\int_{M}\left(\Delta_{g} u_{\epsilon}\right)^{2} d v_{g}=\frac{(n-4) \omega_{n-1} I_{n}^{\frac{n}{2}-1}}{2 \epsilon^{n-4}}\left\{n\left(n^{2}-4\right)-\frac{n\left(n^{2}+4 n-20\right)}{6(n-6)} R\left(x_{0}\right) \epsilon^{2}+o\left(\epsilon^{2}\right)\right\}
$$

and also

$$
\int_{M} A^{\#}\left(d u_{\epsilon}, d u_{\epsilon}\right) d v_{g}=\frac{(n-4) \omega_{n-1} I_{n}^{\frac{n}{2}-1}}{2 \epsilon^{n-4}}\left\{\frac{4(n-1)}{n-6} \operatorname{Tr}_{g} A\left(x_{0}\right) \epsilon^{2}+o\left(\epsilon^{2}\right)\right\} .
$$

And Finally, we have

$$
\int_{M} a u_{\epsilon}^{2} d v_{g}=\frac{1}{\epsilon^{n-4}} O\left(\epsilon^{4}\right) .
$$

Bearing the different terms of $\mu\left(u_{\epsilon}\right)$ in equation (4.1), we obtain

$$
\begin{aligned}
& \mu\left(u_{\epsilon}\right)=\frac{n(n-4)\left(n^{2}-4\right) \omega_{n}}{2^{n} \epsilon^{n-4}} \times \\
& \left\{1+\frac{1}{n\left(n^{2}-4\right)(n-6)}\left(4 \operatorname{Tr}_{g} A\left(x_{0}\right)(n-1)-\frac{n\left(n^{2}+4 n-20\right)}{6} R\left(x_{0}\right)\right) \epsilon^{2}+o\left(\epsilon^{2}\right)\right\}
\end{aligned}
$$

where $\omega_{n}=2^{n-1} I_{n}^{\frac{n}{2}-1} \omega_{n-1}$.

The Taylor's expansion of $\gamma\left(u_{\epsilon}\right)$ is given by

$\gamma\left(u_{\epsilon}\right)=\int_{M} f\left|u_{\epsilon}\right|^{2^{\sharp}} d v_{g}=\frac{f\left(x_{0}\right) \omega_{n}}{2^{n} \epsilon^{n}}\left\{1-\frac{1}{6(n-2)}\left(\frac{3 \Delta f\left(x_{0}\right)}{f\left(x_{0}\right)}+R\left(x_{0}\right)\right) \epsilon^{2}+o\left(\epsilon^{2}\right)\right\}$.

Consequently

$$
\left(\gamma\left(u_{\epsilon}\right)\right)^{-\frac{2}{2^{\sharp}}}=\frac{\left(f\left(x_{0}\right)\right)^{-\frac{2}{2^{\sharp}}} \omega_{n}^{-\frac{2}{2^{\sharp}}}}{2^{4-n} \epsilon^{4-n}}\left\{1+\frac{n-4}{6 n(n-2)}\left(R\left(x_{0}\right)+\frac{3 \Delta f\left(x_{0}\right)}{f\left(x_{0}\right)}\right) \epsilon^{2}+o\left(\epsilon^{2}\right)\right\} .
$$


Therefore the Taylor's expansion of $Q_{\epsilon}$, is given by

$$
\begin{aligned}
Q_{\epsilon} & =\frac{1}{\left(f\left(x_{0}\right)\right)^{-\frac{2}{2^{\sharp}}} K_{0}}\left\{1+\frac{1}{2 n\left(n^{2}-4\right)(n-6)} \times\right. \\
& \left.\left((n+2)(n-4)(n-6) \frac{\Delta f\left(x_{0}\right)}{f\left(x_{0}\right)}+8(n-1) \operatorname{Tr}_{g} A\left(x_{0}\right)-4\left(n^{2}-2 n-4\right) R\left(x_{0}\right)\right) \epsilon^{2}+o\left(\epsilon^{2}\right)\right\}
\end{aligned}
$$

where

It is obvious that if

$$
\frac{1}{K_{0}}=\frac{n(n-4)\left(n^{2}-4\right) \omega_{n}^{\frac{4}{n}}}{16}
$$

$(n+2)(n-4)(n-6) \frac{\Delta f\left(x_{0}\right)}{f\left(x_{0}\right)}+8(n-1) \operatorname{Tr}_{g} A\left(x_{0}\right)-4\left(n^{2}-2 n-4\right) R\left(x_{0}\right)<0$

we have

$$
Q_{\epsilon}<1 \text {. }
$$

In the case $n=6$, we have

$$
\int_{M}\left(\Delta_{g} u_{\epsilon}\right)^{2} d v_{g}=\frac{(n-4)^{2} \omega_{n-1}}{2 \epsilon^{n-4}}\left\{\frac{n\left(n^{2}-4\right)}{n-4} I_{n}^{\frac{n}{2}-1}-\frac{2}{n} R\left(x_{0}\right) \epsilon^{2} \ln \frac{1}{\epsilon^{2}}+o\left(\epsilon^{2}\right)\right\}
$$

and also

$$
\int_{M} A^{\#}\left(d u_{\epsilon}, d u_{\epsilon}\right) d v_{g}=\frac{(n-4)^{2} \omega_{n-1} I_{n}^{\frac{n}{2}-1}}{2 \epsilon^{n-4}}\left(\frac{\operatorname{Tr}_{g} A\left(x_{0}\right)}{n} \epsilon^{2} \ln \frac{1}{\epsilon^{2}}+o\left(\epsilon^{2}\right)\right) .
$$

The expression of the last term is written as

$$
\int_{M} a u_{\epsilon}^{2} d v_{g}=\frac{1}{\epsilon^{n-4}} O\left(\epsilon^{4}\right)
$$

Inserting the different terms of $\mu\left(u_{\epsilon}\right)$ in equation (4.1), we get

$$
\begin{aligned}
\mu\left(u_{\epsilon}\right) & =\int_{M}\left(\left(\Delta_{g} u_{\epsilon}\right)^{2}+A^{\#}\left(d u_{\epsilon}, d u_{\epsilon}\right)+a u_{\epsilon}^{2}\right) d v_{g} \\
& =\frac{n(n-4)\left(n^{2}-4\right) \omega_{n}}{2^{n} \epsilon^{n-4}}\left\{1+\frac{n-4}{\left(n^{2}-4\right) I_{n}^{\frac{n}{2}-1}}\left(\operatorname{Tr}_{g} A\left(x_{0}\right)-2 R\left(x_{0}\right)\right) \epsilon^{2} \ln \frac{1}{\epsilon^{2}}+o\left(\epsilon^{2}\right)\right\}
\end{aligned}
$$

where $\omega_{n}=2^{n-1} I_{n}^{\frac{n}{2}-1} \omega_{n-1}$.

In the same way as in the previous case, we obtain:

$\left(\gamma\left(u_{\epsilon}\right)\right)^{-\frac{2}{2^{\sharp}}}=\frac{\left(f\left(x_{0}\right)\right)^{-\frac{2}{2^{\sharp}}} \omega_{n}^{-\frac{2}{2^{\sharp}}}}{2^{4-n} \epsilon^{4-n}}\left\{1+\frac{n-4}{6 n(n-2)}\left(R\left(x_{0}\right)+\frac{3 \Delta f\left(x_{0}\right)}{f\left(x_{0}\right)}\right) \epsilon^{2}+o\left(\epsilon^{2}\right)\right\}$.

Finally, the Taylor expansion of $Q_{\epsilon}$, when $n=6$, is given by

$Q_{\epsilon}=\frac{1}{\left(f\left(x_{0}\right)\right)^{-\frac{2}{2^{\sharp}}} K_{0}}\left\{1+\frac{n-4}{\left(n^{2}-4\right) I_{n}^{\frac{n}{2}-1}}\left(\operatorname{Tr}_{g} A\left(x_{0}\right)-2 R\left(x_{0}\right)\right) \epsilon^{2} \ln \frac{1}{\epsilon^{2}}+o\left(\epsilon^{2}\right)\right\}$.

Assuming

$$
\operatorname{Tr}_{g} A\left(x_{0}\right)<2 R\left(x_{0}\right)
$$


we get

$$
Q_{\epsilon}<1 .
$$

\section{REFERENCES}

[1] F. Atkinson, H. Brezis, L. Peletier, Nodal solutions of elliptic equations with critical Sobolev exponents, J. Differential Equations 85 (1990), 151170.

[2] T. Aubin, Equations différentielles non linéaires et problème de Yamabe concernant la courbure scalaire, J. Math. Pures Appl. 55 (1976) 269-296.

[3] T. Aubin, Some nonlinear problems in Riemannian geometry, Springer (1998).

[4] M. Benalili. On singular Q-curvature type equations, J. Differential Equations, 254, 2, (2013) 547-598.

[5] T.P. Branson, Group representations arising from Lorentz conformal geometry, J. Funct. Anal. 74, 1987, 199-291.

[6] R. J. Biezuner, M. Montenegro, Best constants in second-order Sobolev inequalities on Riemannian manifolds and applications, J. Math. Pures Appl. 82 (2003), 457-502.

[7] D. Caraffa, Equations elliptiques du quatrième ordre avec exposants critiques sur les variétés riemanniennes compactes. J. Math. Pures Appl., 80, 9 (2001), 941-960.

[8] S.Y.A. Chang. On Paneitz operator, A fourth order differential operator in conformal geometry, Harmonic Analysis and PDE, Essays in honor of Alberto P. Calderon, Eds: M. Christ, C. Kenig and C. Sadorsky, Chicago Lectures in Mathematics, 1999, 127150.

[9] Chang. S.Y.A, Yang. P.C., On a fourth order curvature invariant, Comp. Math. 237, Spectral Problems in Geometry and Arithmetic, Ed: T. Branson, AMS, 1999, 9-28.

[10] Z. Djadli, E. Hebey, M. Ledoux, Paneitz-type operators and applications. Duke Math. J. 104, (2000), 129-169.

[11] Z. Djadli, A. Jourdain, Nodal solutions for scalar curvature type equations with perturbation terms on compact Riemannian manifolds. Boll. Unione Mat. Ital. Sez. Artic. Ric. Mat. (8), 5 (2002), No 1, 205-226.

[12] Z. Djadli, A. Malchiodi and M. Ould Ahmedou. Prescribed fourth order conformal invariant on the standard sphere - Part I: a perturbation result, Commun. Contemp. Math. 04, 375 (2002).

[13] Z. Djadli, A. Malchiodi and M. Ould Ahmedou. Prescribed fourth order conformal invariant on the standard sphere - Part II: blow-up analysis and applications, Ann. Scuola Norm. Sup. Pisa, Vol. I (2002), pp. 387-434.

[14] D.E. Edmunds, D. Fortunato, E.Jannelli, Critical exponents, critical dimensions and the biharmonic operator. Arch. Rational Mech. Anal., 112, (1990), no. 3, 269-289.

[15] P. Esposito, F. Robert. Mountain pass critical points for Paneitz-Branson operators. Calc. Var. Partial Differential Equations, 15, (2002), 493-517.

[16] V. Felli. Existence of conformal metrics on $S_{n}$ with prescribed fourth order invariant, Adv. Differential Equations, 7, 2002, 47-76.

[17] E. Hebey and F. Robert. Coercivity and Struwe's compactness for Paneitz type operators with constant coefficients, Calc. Var. Partial Differential Equations, 13, 2001, 491-517.

[18] E. Hebey and M. Vaugon. Existence and multiplicity of nodal solutions for nonlinear elliptic equations with critical Sobolev growth, J. Funct. Anal. 119(2) (1994), 298-318.

[19] D. Holcman. Solutions nodales sur les variétés Riemanniennes non localement conformément plates à bord Comment. Math. Helv. 76 (2001) 373-387

[20] E.H. Lieb. Sharp constants in the Hardy-Littlewood-Sobolev and related inequalities. Ann. of Math., (2), 118, (1983), no. 2, 349-374.

[21] Lin, C.S., A classification of solutions of a conformally invariant fourth order equation in $\mathbb{R}^{n}$, Comment. Math. Helv., 73, 1998, 206-231. 
[22] P.L. Lions. The concentration-compactness principle in the calculus of variations. The limit case. I and II. Rev. Mat. Iberoamericana, 1, (1985), no. 1, 145-201, no. 2, 45-121.

[23] S. Paneitz. A quatric conformally covariant differential operator for arbitrary peudoRiemannian manifolds, SIGMA, 4, (2008).

[24] F. Robert. Positive solutions for a fourth order equation invariant under isometries, Proc. Amer. Math. Soc, (2002), no 5, 1423-1431.

[25] F. Robert. Fourth order equations with critical growth in Riemannian geometry. Notes from a course given at the University of Wisconsin at Madison and at Technische Universitat in Berlin.

[26] R.C.A.M. Van der Vorst. Best constant for the embedding of the space $H^{2} \cap H_{0}^{1}(\Omega)$ into $L^{\frac{2 N}{N-4}}(\Omega)$. Differential Integral Equations, 6, (1993), 259-276.

[27] H. YAMABE, On the deformation of Riemannian structures on compact manifolds, Osaka Math. J. 12 (1960), 21-37.

Mohamed Bekiri Faculty Of Sciences, Mathematics Dept; University AbouBakr Belkaîd; Tlemcen. Algeria

E-mail address: bekiri03@yahoo.fr

Mohammed Benalili Faculty Of Sciences, Mathematics Dept; University Abou-Bakr Belkaîd; Tlemcen. Algeria

E-mail address: m_benalili@mail.univ-tlemcen.dz 